\title{
New Method for Producing Graphene by Magnetron Discharge in an Atmosphere of Aromatic Hydrocarbons
}

\author{
Bagila Baitimbetova, Boris Vermenichev \\ Kazakh National Technical University after K.I. Satpaev Almaty, Almaty, Republic of Kazakhstan \\ Email: baitim@physics.kz
}

Received 10 April 2015; accepted 20 April 2015; published 23 April 2015

Copyright (C) 2015 by authors and Scientific Research Publishing Inc.

This work is licensed under the Creative Commons Attribution International License (CC BY). http://creativecommons.org/licenses/by/4.0/

(c) (i) Open Access

\begin{abstract}
This paper proposes the method of producing graphene sheets and carbon nanotubes with reactive magnetron sputtering in vapour by sublimation aromatic hydrocarbons (naphthalene) with a structure of the benzene rings in a more natural formation of graphene structures. The carbon grid of molecular structure of aromatic hydrocarbons coincides with the graphene of carbon grid. The article shows the method of obtaining carbon nanostructures. The graphene of peaks was observed with the vibrational mode (2D-zone) at a frequency of $\sim 2728 \mathrm{sm}^{-1}$ using the method of Raman spectroscopy. Results from studies using atomic force microscopy confirm the formation of graphene sheets and the carbon nanotubes.
\end{abstract}

\section{Keywords}

Aromatic Hydrocarbons, Graphene, Carbon Nanotube, Magnetron Sputtering, Naphthalene

\section{Introduction}

Graphene has some interesting properties that allow us to consider it as a promising material for nanoelectronics, optoelectronics and other applications [1] [2]. The magnetron sputtering of carbon film plays an important role in the nanotechnology for producing new thin film materials, including nanotubes. It is based on the chemical processes to decompose gaseous compounds in the impact of plasma ions. The film is formed at low temperatures using parameters of the glow discharge (the vapor pressure, the discharge current and voltage). It allows us to change the condensation conditions, the composition, the packing density of the atoms, and the physical properties of the films in a broad range [3].

Nowadays, a topical problem in the modern microelectronics is preparing the graphene films of large sizes of 
and other nanostructures, and their sedimentation on the surface. A wide range of methods production allows us to obtain nanostructure such as thin films, nanotubes, graphene, anions, fullerenes. Among them the plasma technology is a simple and economical method. This technology plays an important role in obtaining the required products for use in the science and technology. This paper examines the significance of plasma technology in the process of producing thin carbon films. The plasma nanotechnology creates the graphite films, nanotubes, and other nanostructures based on them, which is an essential part of magnetron of the technology [4]. Carbon films are successfully used as anti-reflection coatings and in nanoelectronics and optical devices. This is due to their different properties, such as dielectric properties, thermal conductivity, high mechanical strength and etc.

Nanocarbon structures are obtained through magnetron sputtering, which is one of the methods of producing nanocarbon structures in the form of carbon nanotubes. In our view, this method is very interesting. This method is disclosed by the authors in patent and paper [5] [6]. We have chosen this method as a basis for a further ransformation to obtain graphene structures, not only by the selection of substrates, but also in the respective terms of the magnetron discharge.

In this paper, a new technique of obtaining graphene structures for producing the carbon films is considered, which are formed on the simplest, reactive magnetron sputtering of graphite in an atmosphere of sublime vapor of aromatic hydrocarbons [7].

\section{Experimental Part}

The method of magnetron sputtering is a well-known traditional methods for producing thin film carbon structures. The first step in this process is to introduce the reactive gas, such as methane, through the inlet valve to the gas from a gas cylinder in the working chamber of the vacuum unit, where the introduced gas is mixed with the basic argon gas, to create a favorable environment for the synthesis of carbon film structures. The second step is to state the observed pressure in the working chamber.

The pressure was $5 \times 10^{-3}$ Torr in the presence of aromatic hydrocarbons in the chamber, the pressure in the chamber installed $6 \times 10^{-2}$. Torr after the launch of the argon, the sputtering material was produced at a current of $150 \mathrm{~mA}$ and a voltage of $200-300 \mathrm{~V}$ (Figure 1, Figure 2). The following three substrates were used: aluminum, glass and stainless steel X18H10T. The substrates were attached to the duralumin table, at a distance of 3 $5 \mathrm{~cm}$ above the target. Sputtering was carried out from 20 minutes to 1 hour (Figures 1-3).

The advantage of the new method of is producing graphene structures, nanowires and nanotubes are the using of aromatic hydrocarbons such as naphthalene, phenanthrene, and others with a close affinity to graphene structures.

In this method is utilizes the ability of the vapor aromatic hydrocarbons which to sublimate an ambient temperatures and reacts as reactive magnetron sputtering gas.

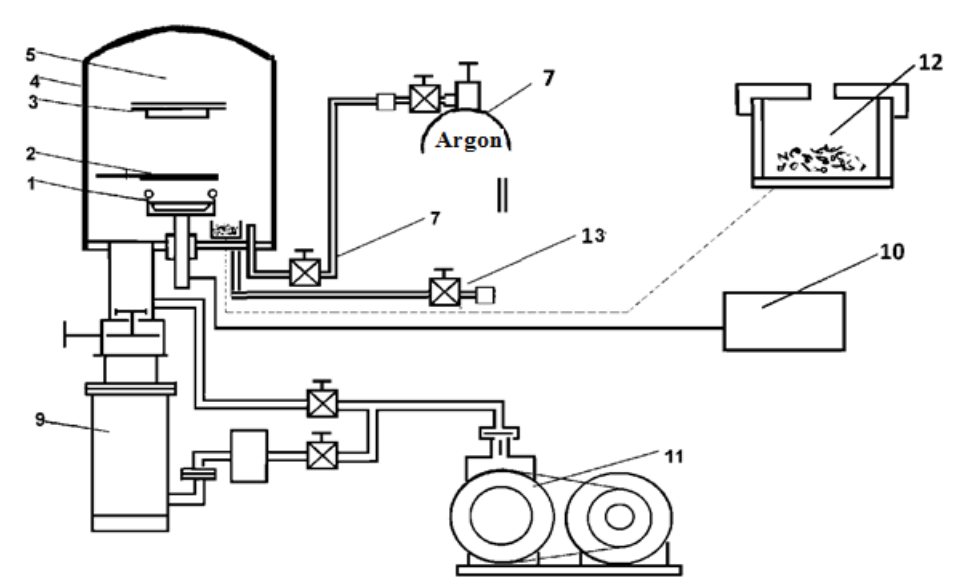

1-graphite target, 2-damper, 3-a sample holder (substrate) 4-duralumin smal chair 5the working vacuum chamber,, 6-leak valve, 7-and argon balloon, 8-cylinder gases (for conventional methods of methane gas) 9-diffusion pump, 10-high-voltage power supply, 11-backing pump, 12-case for the sublime substances, 13-capacity for liquid aromatics.

Figure 1. Scheme magnetron reactive sputtering in an atmosphere of aromatic hydrocarbons. 


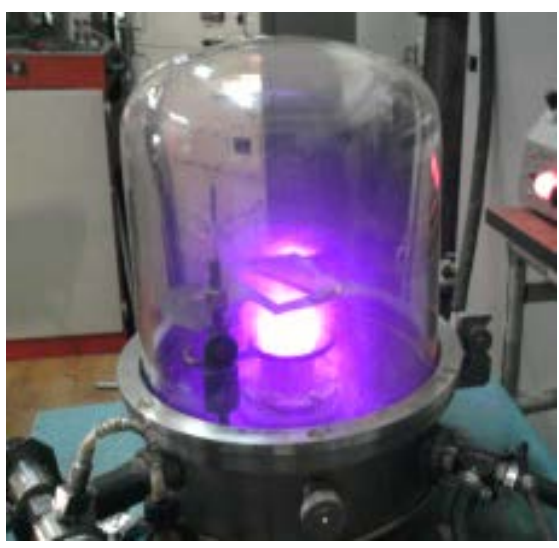

Figure 2. Magnetron discharge in the atmosphere of aromatic hydrocarbons.

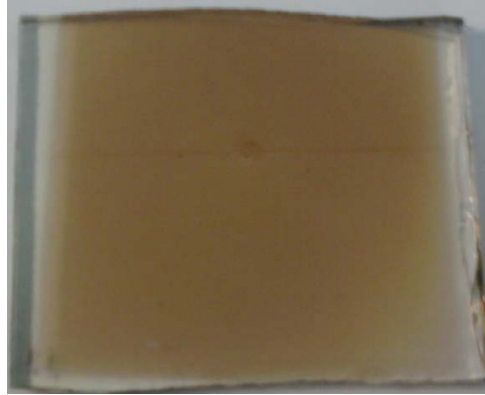

(a)

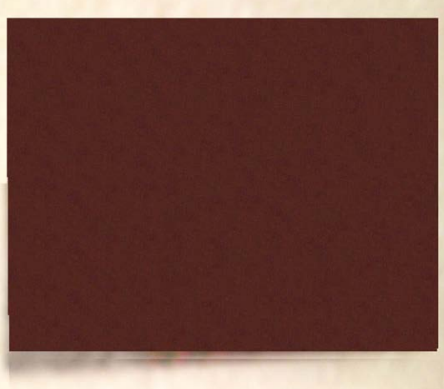

(b)

Figure 3. An area of the sputtered film on different substrates approximately 6 $\times 7 \mathrm{~cm}$. (a) Glasse; (b) Stainless steel X18H10T.

The cathode is a graphite disk which is separated from the cooling water chamber with aluminum foil to prevent leakage of cooling water through the pores of the graphite cathode.

The peculiarity in this method of obtaining graphene structures is that the aromatic hydrocarbon, e.g. naphthalene, is placed directly within the vacuum work. Naphthalene $\left(\mathrm{C}_{10} \mathrm{H}_{8}\right)$ is an aromatic compound with a flat carbon skeleton in which all 10 carbon atoms are in $\mathrm{sp}^{2}$-hybridized with the valence angles $120^{\circ}$, pairing covers 2 cycles, the number of electrons involved in conjugation is $10(4 \cdot 2+2=10 \pi$-electrons associated $5 \pi$-bonds $)$ (Figure 4).

The structure of the aromatic hydrocarbon comprises benzene rings surrounded by hydrogen atoms, (which in reactive magnetron discharge are released and carried away by a vacuum pumping system,) and the skeletons of these molecular structures similar to graphene cells.

Depending on the nature of the substrates on which during the sputtering are formed the nanocarbon produces various structures such as the structure of graphene and carbon nanotubes. Analogues of this method are obtaining nanostructured carbon materials hasn't been detected [7].

Traditionally, the morphology and structural properties of carbon films, including graphene structure and carbon nanotubes were assessed by measuring Raman spectroscopy and atomic force microscopy. Microscopic studies were performed on a scanning atomic force microscope (AFM) MT-MDT Integra Prima. The Raman spectra were measured using a MT-MDT Integra Spectra at room temperature. Spectra were excited by a semiconductor laser $(\lambda=473 \mathrm{~nm})$.

\section{Results and Discussion}

In a detailed study, probe atomic force microscopy (AFM) was scanned. This part of the image showed that the glass surface modified stainless steel with a thin layer of carbon. For these values of the thickness the carbon layer is not continuous, but it consists of clusters and nanotubes, graphene structure with dimensions about hundreds of nanometers. The length of graphene ribbons is over 5 microns in Figure 5(a), Figure 5(b). Carbon na- 

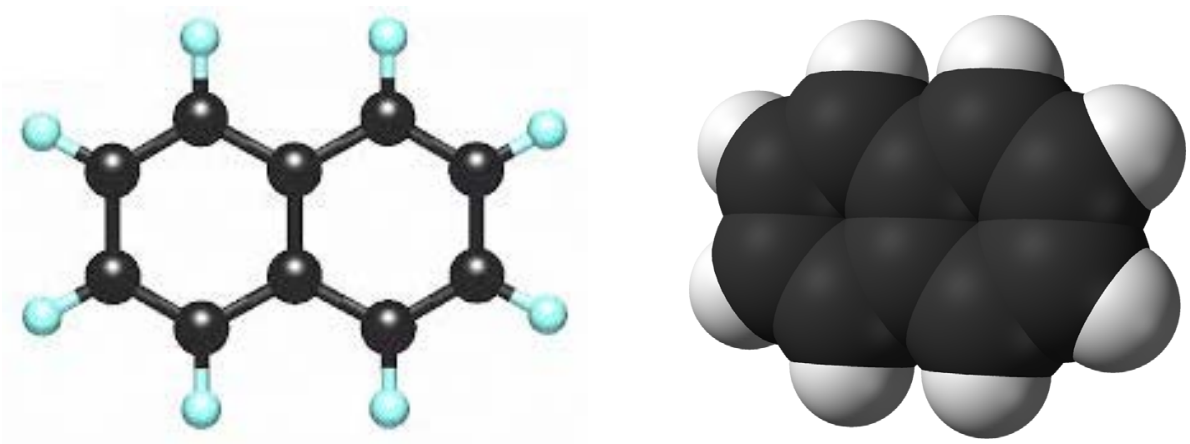

Figure 4. The structure of naphthalene.

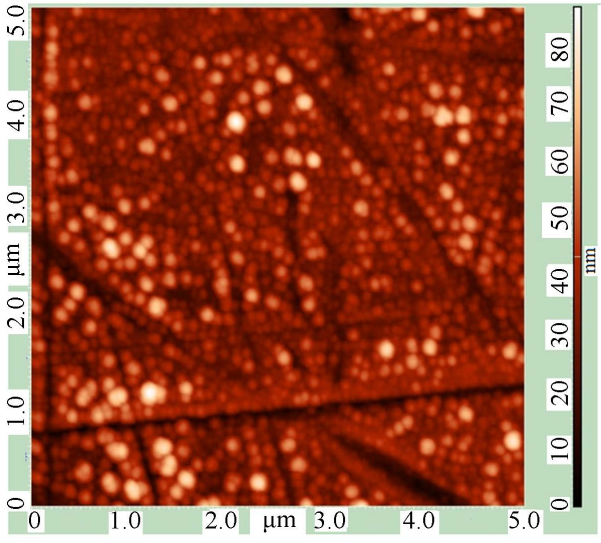

(a)

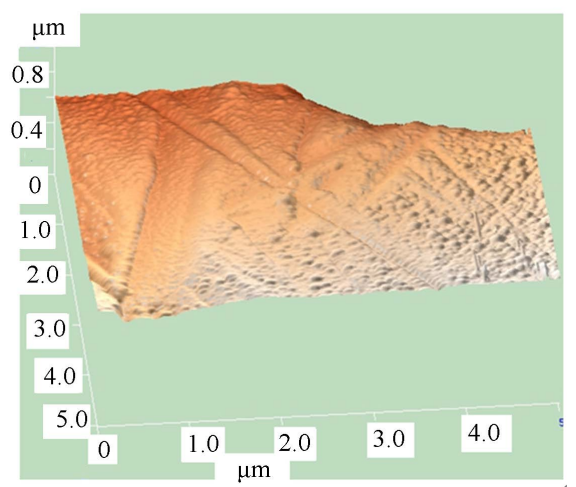

(b)

Figure 5. Images of graphene ribbons using atomic force microscopy on a stainless steel. (a) Two-dimensional image of graphene ribbons; (b) Three-dimensional image of graphene ribbons.

notubes are clearly visible with a length of about $100 \mathrm{~nm}$ to $80 \mathrm{~nm}$ diameters and above. The glass substrate has a tube packing density less than that of stainless steel Figure 6(a), Figure 6(b).

Micrographic images of the surface of the carbon films show that their structure is of tightly focused nanotubes vertically to the substrate surface. More favorable conditions for the formation of graphene and nanotubes on the substrate take place in stainless steel due to the presence of iron. Iron in this case is the catalyst for the formation of graphene sheets and other carbon nanostructures. The surface profile of the plate film is vertical (forest) a carbon nanotube perpendicular to the substrate surface. The section profile shows that the number of nanotubes in the range of 0 to 5 microns is 18 - 20 etc.

Raman spectroscopy is an effective of method to determine the presence of graphene structures. It is known that the shape and intensity of peaks is unambiguously determined by the number of layers in the sample [8][15]. This method gives a lot of information about the vibrational properties and structure of short-range order of carbon materials, which are a useful source of information on the investigated materials [16].

Table 1 shows the parameter of the Raman spectra of graphene samples obtained by magnetron reactive sputtering in an atmosphere of graphite aromatic hydrocarbons. Figure 7 and Figure 8 shows the Raman spectrum for stainless steel X18H10TH1.

Seen from Figure 7 that the spectrum observed G band at area 1350 and $1593 \mathrm{sm}^{-1}$, which are conditioned carbon nanotubes. D band in area $2500-2900 \mathrm{sm}^{-1}$ is conditioned graphene ribbons. The carbon films were measured on a substrate of glass and aluminum using the Raman spectra. Analysis of the spectra of carbon films shows that the number of graphene structures studied depends essentially on the nature of the substrate.

The results showed that the on glass and aluminum have low intensity, and the intensity spectrum of stainless steel has a significantly greater value. This is due to the fact that the composition of stainless steel has iron, which plays an important catalysing role to catalyst the formation of graphene structures. Absence of a catalyst on substrates of glass, aluminum and weakens the intensity of the graphene spectrum. 
Table 1. Parameters of the Raman spectra of carbon films obtained by magnetron discharge with naphthalene.

\begin{tabular}{|c|c|c|c|c|c|c|c|c|}
\hline \multirow{2}{*}{ No. } & \multirow{2}{*}{ Substrate } & \multicolumn{2}{|c|}{ D-line } & \multicolumn{2}{|c|}{ G-line } & \multicolumn{2}{|c|}{ 2D-line } & \multirow{2}{*}{$\mathrm{I}_{2 \mathrm{D}} / \mathrm{I}_{\mathrm{G}}$} \\
\hline & & $\omega, \mathrm{sm}^{-1}$ & FWHM, $\mathrm{sm}^{-1}$ & $\omega, \mathrm{sm}^{-1}$ & FWHM, $\mathrm{sm}^{-1}$ & $\omega, \mathrm{sm}^{-1}$ & FWHM, $\mathrm{sm}^{-1}$ & \\
\hline 1 & Glass & 1341 & 80 & 1580 & 32 & 2797 & 250 & - \\
\hline 2 & Aluminum plate & 1332 & 76 & 1579 & 28 & 2795 & 279 & 0.57 \\
\hline 3 & Stainless steel X18H10T & 1363 & 13 & 1579 & 15 & 2728 & 50 & 0.68 \\
\hline
\end{tabular}

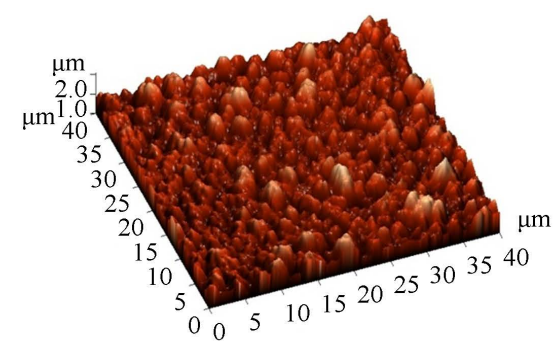

(a)

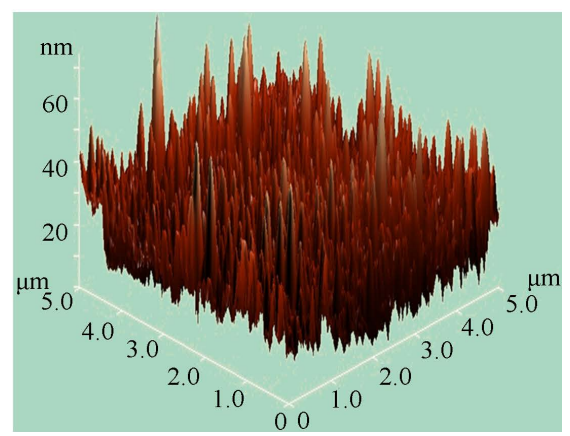

(b)

Figure 6. AFM images of the surface of the carbon films. (a) On a glass substrate; (b) On a stainless steel.

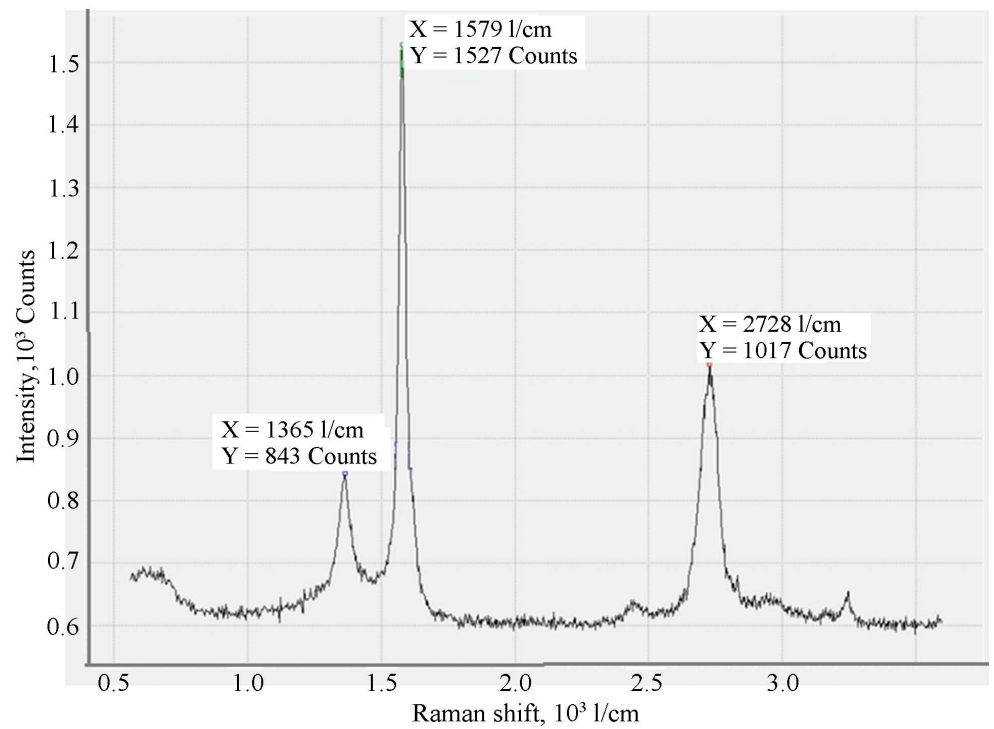

Figure 7. The Raman spectrum of the carbon film on the stainless steel substrate.

\section{Conclusion}

One of the most significant findings to emerge from this study is that the graphene sheets and nanotubes first were produced using reactive magnetron sputtering in the vapor of aromatic hydrocarbons including naphthalene. The results of studies using atomic force microscopy confirm the formation of graphene and carbon nanotubes obtained by this method. The investigation of Raman spectroscopy has shown that the peaks of graphene is observed with the vibrational mode (2D-zone) at a frequency of $\sim 2728 \mathrm{sm}^{-1}$ on a substrate of stainless steel using Raman spectroscopy. The most obvious finding in this study is that the aromatic hydrocarbons, which are used to produce graphene and nanocarbon structures, give impetus to their more efficient obtaining. 


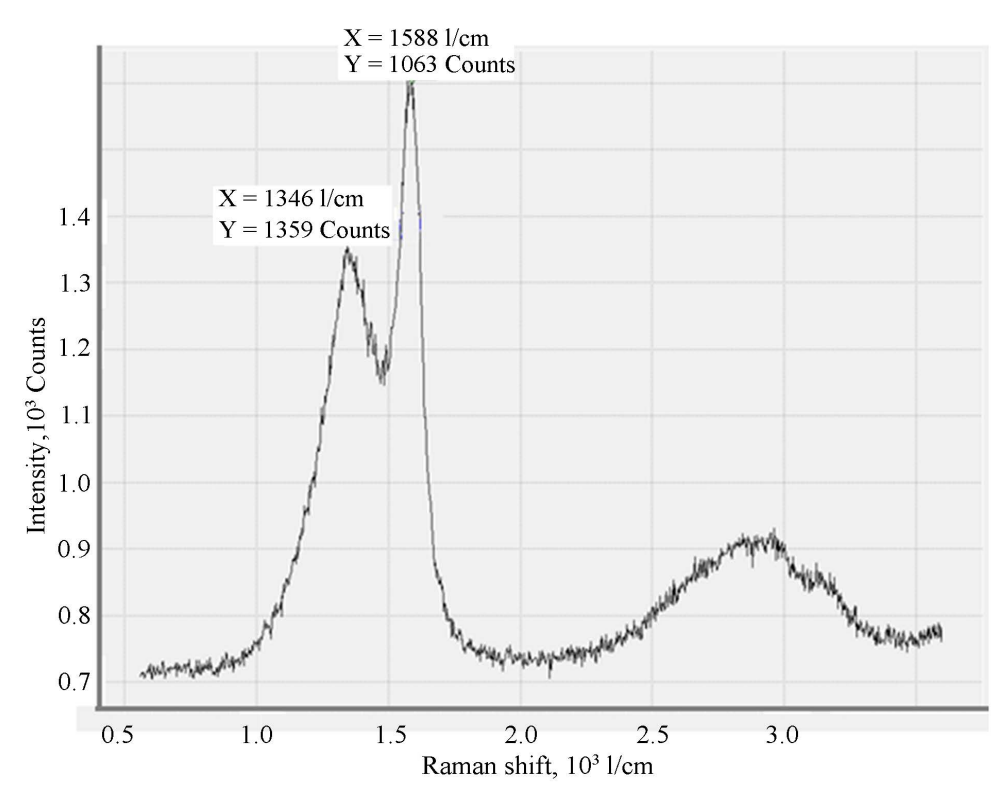

Figure 8. Raman spectrum of the carbon film on a substrate aluminum.

\section{Acknowledgements}

This work was supported by the Ministry of Education and Science of the Republic of Kazakhstan under grant No. 751. MES. GF.12.7.

\section{References}

[1] Geim, A.K. and Novoselov, K.S. (2007) The Rise of Graphene. Nature Materials, 3, 183-191. http://dx.doi.org/10.1038/nmat1849

[2] Sorokin, P.B. and Chernozatonskii, L.A. (2013) Semiconductor Nanostructures Based on Graphene. Physics-Uspekhi, 2, 113-132. http://dx.doi.org/10.3367/UFNr.0183.201302a.0113

[3] Dekoven, B.M., Ward, P.R., Weiss, R.E., et al. (2003) Carbon Thin Film Deposition Using High Power Pulsed Magnetron Sputtering. The 46th Annual Technical Conference Proceedings, 158-165.

[4] Cashtanov, P.V., Smirnov, B.M. and Hippler, R. (2007) Magnetron Plasma and Nanotechnology. Physics-Uspekhi, 5, 473-51.

[5] Antonenko, S.V. and Maltsev, S.N. (2005) Preparation of Carbon Nanotubes by Magnetron Sputtering. Patent No. 2355625.

[6] Antonenko, S.V. and Maltsev, S.N. (2005) Preparation of Carbon Nanotubes by Magnetron Sputtering with Direct Current. Instruments and Experiment, 3, 150-152.

[7] Baitimbetova, B.A. and Vermenichev, B.M. (2013) A Method for Producing Carbon Nanostructures by Magnetron Reactive Sputtering of Graphite in Sublime Pairs of Aromatic Hydrocarbons. Patent RK, 0803.1.

[8] Liu, Y., Pan, C. and Wang, J. (2004) Raman Spectra of Carbon Nanotubes and Nanofibers Prepared by Ethanol Flames. Journal of Materials Science, 39, 1091-1094. http://dx.doi.org/10.1023/B:JMSC.0000012952.20840.09

[9] Eklud, P.C., Holden, J.M. and Jishi, R.A. (1995) Vibrational Modes of Carbon Nanotubes; Spectroscopy and Theory. Carbon, 33, 959-972. http://dx.doi.org/10.1016/0008-6223(95)00035-C

[10] Nemanich, R.J. and Solin, S.A. (1979) First- and Second-Order Raman Scattering from Finite-Size Crystals of Graphite. Physics Review, B20, 392-401. http://dx.doi.org/10.1103/PhysRevB.20.392

[11] Ferrari, A.C. (2007) Raman Spectroscopy of Graphene and Graphite: Disorder, Electron-Phonon Coupling, Doping and Nonadiabatic Effects. Solid State Communications, 143, 47-57.

[12] Ni, Z., Wang, Y., Yu, T. and Shen, Z. (2008) Raman Spectroscopy and Imaging of Graphene. Nano Research, 1, 273291.

[13] Mohiuddin, T.M.G., Lombardo, A., Nair, R.R., Bonett, A., Savini, G., Jalil, R., Bonini, N., Basko, D.M. and Galiotis, C. (2009) Uniaxial Strain in Graphene by Raman Spectroscopy: G Peak Splitting, Grueneisen Parameters, and Sample 
Orientation. Physical Review B (Condensed Matter and Materials Physics), 79, Article ID: 205433.

[14] Ferrari, A.C., Meyer, J.C., Scardaci, V., Casiraghi, C., Lazzeri, M., et al. (2006) Raman Spectrum of Graphene and Graphene Layers. Physical Review Letters, 97, Article ID: 187401.

[15] Luican, A., Li, G.H., Reina, A., Kong, J., Nair, R.R., Novoselov, K.S., Geim, A.K. and Andrei, E.Y. (2011) Single-Layer Behavior and Its Breakdown in Twisted Graphene Layers. Physical Review Letters, 106, Article ID: 126802.

[16] Reich, S. and Thomsen, Ch. (2004) Raman Spectroscopy of Graphite. Philosophical Transactions of the Royal Society of London, 2271-2288. 